\title{
ON THE INVERSION OF THE GAUSS TRANSFORMATION
}

\author{
P. G. ROONEY
}

1. Introduction. The inversion theory of the Gauss transformation has been the subject of recent work by several authors. If the transformation is defined by

$$
f(x)=\mathscr{G}(\phi(x))=(4 \pi)^{-\frac{1}{2}} \int_{-\infty}^{\infty} e^{-\frac{1}{4}(x-t)^{2}} \phi(t) d t,
$$

then operational methods indicate that

$$
\exp \left(-D^{2}\right) f^{\prime}(x)=\phi(x),
$$

under a suitable definition of the differential operator. Hirschmann and Widder (5; chap. 8) have shown that this is indeed the case if the operator is defined by use of the formula

$$
e^{-y^{2}}=\lim _{t \rightarrow 1-} \frac{1}{2 \pi i} \int_{a-i_{\infty}}^{a+i_{\infty}} K(s, t) e^{s y} d s,
$$

where

$$
K(s, t)=(\pi / t)^{\frac{1}{2}} \exp \left(s^{2} / 4 t\right) .
$$

Also Widder $(8 ; \S 1)$ makes use of the formula

$$
e^{-y^{2}}=\pi^{-\frac{1}{2}} \int_{0}^{\infty} e^{-\frac{1}{t} t^{2}} \cos y t d t
$$

to define $\exp \left(-D^{2}\right)$.

A more straightforward approach seems to be to use the power series for

$$
e^{-y^{2}}
$$

This we shall do here, defining

$$
e^{-D^{2}} f(x)=\sum_{n=0}^{m}(-1)^{n} f^{(2 n)}(x) / n !
$$

This approach seems to have been originally suggested by Eddington (2). Eddington did not study the convergence of this series other than to note that for some very simple Gauss transforms, the series diverges. Widder (8; $\S 1$ ) remarks that for some very simple transforms the series is not even summable in the Abel sense. Pollard $(6 ; \S 8)$ showed that if $\phi \in L_{2}(-\infty, \infty)$, the series converges in the $L_{2}$-sense to $\phi$, that is

Received September 13, 1956; in expanded form October 3, 1956. 


$$
\phi(x)=\underset{r \rightarrow \infty}{\lim . \mathrm{m}} \sum_{n=0}^{r}(-1)^{n} f^{(2 n)}(x) / n !,
$$

but nothing seems to be known of the pointwise convergence of the series.

Here we propose to give sufficient conditions for the series to converge and to be Abel summable to $\phi(x)$. This occupies $\$ \S$ two and three respectively. In $\S$ four we make some applications of our results to the study of Hermite polynomials.

2. Convergence of the series. Theorem 1 gives sufficient conditions for the series II to converge at a point to the mean value of $\phi$ there.

Theorem 1. If

$$
\phi \in L(-\delta, \delta) \quad \delta>0 \text {, and }|t|^{\lambda} e^{-\left(x_{0}-t\right)^{2} / 8} \phi(t) \in L(-\infty, \infty),
$$

for some $\lambda>3$,

(ii) $\phi$ is of bounded variation in a neighbourhood of $x_{0}$, then $\mathscr{Y}(\phi(x))$ exists for all $x$, and the series 1.2 converges at $x=x_{0}$ to $\frac{1}{2}\left\{\phi\left(x_{0}+\right)+\phi\left(x_{0}-\right)\right\}$.

Proof. The existence of $\mathscr{G}(\phi(x))$ is clear. Now by $(3 ; 10.13(7)$ and $10.13(2))$,

$$
D^{2 r} e^{-\frac{1}{4} x^{2}}=2^{-2 r} e^{-\frac{1}{2} x^{2}} H_{2 r}\left(\frac{1}{2} x\right)=e^{-\frac{1}{4} x^{2}} r !(-1)^{r} L_{r}^{-\frac{1}{2}}\left(\frac{1}{4} x^{2}\right) .
$$

But since $f(x)$ is a bilateral Laplace transform, it follows from (1; chap. 3 $\S 2$ ) that we may differentiate the integral for $f$ under the integral sign as often as we choose. Thus

$$
f^{(2 r)}(x)=\frac{r !(-1)^{r}}{(4 \pi)^{\frac{1}{2}}} \int_{-\infty}^{\infty} e^{-\frac{1}{4}(x-t)^{2}} L_{r}^{-\frac{1}{2}}\left(\frac{1}{4}(x-t)^{2}\right) \phi(t) d t .
$$

Hence if $s_{n}(x)$ denotes the $n$th partial sum of the series 1.2 , we have, using $(3 ; 10.12(38))$,

$$
\begin{aligned}
& s_{n}\left(x_{0}\right)=\sum_{r=0}^{n}(-1)^{r} f^{(2 r)}\left(x_{0}\right) / r \text { : } \\
& =\frac{1}{(4 \pi)^{\frac{1}{3}}} \int_{-\infty}^{\infty} e^{-\frac{1}{4}\left(x_{0}-t\right)^{2}} \sum_{r=0}^{n} L_{r}^{-\frac{1}{2}}\left(\frac{1}{4}\left(x_{0}-t\right)^{2}\right) \phi(t) d t \\
& =\left(\int_{-\infty}^{x_{0}-n^{1 / 2 \lambda}}+\int_{x_{0}-n^{1 / 2 \lambda}}^{x_{0}+n^{1 / 2 \lambda}} \int_{x_{0}+n^{1 / 2 \lambda}}^{\infty}\right) L_{n}^{\frac{1}{2}}\left(\frac{1}{4}\left(x_{0}-t\right)^{2}\right) \phi(t) d t \\
& =I_{1}+I_{2}+I_{3} \text {. }
\end{aligned}
$$

Consider $I_{3}$. By $(3 ; 10.18(14))$,

$$
e^{-\frac{1}{2} x}\left|L_{n}^{\alpha}(x)\right| \leqslant \Gamma(\alpha+1+n) / n ! \Gamma(\alpha+1), \quad \alpha \geqslant 0 .
$$

Hence

$$
\begin{aligned}
\left|I_{3}\right| & \leqslant \frac{\Gamma(n+3 / 2)}{\Gamma(3 / 2) n !(4 \pi)^{\frac{3}{2}}} \int_{x_{0}+n^{1 / 2 \lambda} e^{-\left(x_{0}-t\right)^{2} / 8}|\phi(t)| d t}^{\infty} \\
& \leqslant A \frac{\Gamma(n+3 / 2)}{n !\left(x_{0}+n^{1 / 2 \lambda}\right)^{\lambda}} \int_{x_{0}+n^{1 / 2 \lambda} t^{\lambda} e^{-\left(x_{0}-t\right)^{2} / 8}|\phi(t)| d t .}^{\infty}
\end{aligned}
$$


But from Stirling's formula, $\Gamma(n+3 / 2) / n !=O\left(n^{\frac{1}{2}}\right)$ as $n \rightarrow \infty$, and thus

$$
\Gamma(n+3 / 2) / n !\left(x_{0}+n^{\frac{1}{2} \lambda}\right)^{\lambda}=O(1) \quad \text { as } n \rightarrow \infty,
$$

so that $I_{3}=o(1)$ as $n \rightarrow \infty$. Similarly $I_{1}=o(1)$ as $n \rightarrow \infty$.

Now by $(3 ; 10.15(2))$ and using Stirling's formula, since $\lambda>3$,

$$
L_{n}^{\frac{1}{2}}\left(\frac{1}{4} x^{2}\right) \sim(2 / \pi)^{\frac{1}{2}} e^{x^{2} / 8} \sin \left((n+3 / 4)^{\frac{1}{2}} x\right) / x
$$

as $n \rightarrow \infty$, uniformly in $-n^{1 / 2 \lambda} \leqslant x \leqslant n^{1 / 2 \lambda}$. Hence

$$
\begin{aligned}
I_{2} \sim & \frac{1}{\pi} \int_{x_{0}-n^{1 / 2 \lambda}}^{x_{0}+n^{1 / 2 \lambda}} e^{-\left(x_{0}-t\right)^{2} / 8} \frac{\sin \left((n+3 / 4)^{\frac{1}{2}}\left(x_{0}-t\right)\right)}{x_{0}-t} \phi(t) d t \\
& =\int_{-\infty}^{\infty}-\int_{-\infty}^{x_{0}-n^{1 / 2 \lambda}}-\int_{x_{0}+n^{1 / 2 \lambda}}^{\infty}=I_{4}+I_{5}+I_{6} .
\end{aligned}
$$

For $I_{6}$ we have if $n>0$,

$$
\left|I_{6}\right| \leqslant \int_{x_{0}+n^{1 / 2 \lambda}}^{\infty} e^{-\left(x_{0}-t\right)^{2} / 8} \phi(t) /\left(t-x_{0}\right) d t,
$$

so that $I_{6}=o(1)$ as $n \rightarrow \infty$, and similarly $I_{5}=o(1)$ as $n \rightarrow \infty$.

But by the Fourier integral theorem,

$$
I_{4} \rightarrow \frac{1}{2}\left\{\phi\left(x_{0}+\right)+\phi\left(x_{0}-\right)\right\},
$$

so that

$$
\lim _{n \rightarrow \infty} s_{n}\left(x_{0}\right)=\frac{1}{2}\left\{\phi\left(x_{0}+\right)+\phi\left(x_{0}-\right)\right\},
$$

and the theorem is proved.

\section{Abel summability of the series.}

THEOREM 2. If

$$
\begin{aligned}
& \text { 1. } e^{-\left(x_{0}-t\right)^{2} / 8} \phi(t) \in L(-\infty, \infty), \\
& \text { 2. } \phi\left(x_{0}+\right) \text { and } \phi\left(x_{0}-\right) \text { exist, }
\end{aligned}
$$

then $\mathscr{G}(\phi(x))$ exists for all $x$, and the series II is Abel summable for $x=x_{0}$ to $\frac{1}{2}\left\{\phi\left(x_{0}+\right)+\phi\left(x_{0}-\right)\right\}$.

Proof. The existence of $\mathscr{G}(\phi(x))$ is clear. If

$$
u(x, t)=\sum_{r=0}^{\infty}(-t)^{r} f^{(2 r)}(x) / r !, \quad 0 \leqslant t<1,
$$

we must show that $u\left(x_{0}, 1-\right)=\frac{1}{2}\left\{\phi\left(x_{0}+\right)+\phi\left(x_{0}-\right)\right\}$. But as in the proof of Theorem 1,

$$
f^{(2 r)}(x)=\frac{r !(-1)^{r}}{(4 \pi)^{\frac{1}{2}}} \int_{-\infty}^{\infty} e^{-\frac{1}{4}(x-y)^{2}} L_{r}^{-\frac{1}{2}}\left(\frac{1}{4}(x-y)^{2}\right) \phi(y) d y .
$$

so that using $(3 ; 10.12(17))$, if $0 \leqslant t<1$, 


$$
\begin{aligned}
u\left(x_{0}, t\right) & =(4 \pi)^{-\frac{1}{2}} \sum_{r=0}^{\infty} t^{r} \int_{-\infty}^{\infty} e^{-\frac{1}{4}\left(x_{0}-y\right)^{2}} L_{r}^{-\frac{1}{2}}\left(\frac{1}{4}\left(x_{0}-y\right)^{2}\right) \phi(y) d y \\
& =\frac{1}{(4 \pi)^{\frac{1}{2}}} \int_{-\infty}^{\infty} e^{-\frac{1}{4}\left(x_{0}-y\right)^{2}} \sum_{r=0}^{\infty} t^{r} L_{r}^{-\frac{1}{2}}\left(\frac{1}{4}\left(x_{0}-y\right)^{2}\right) \phi(y) d y \\
& =\frac{1}{(4 \pi(1-t))^{\frac{3}{2}}} \int_{-\infty}^{\infty} e^{-\left(x_{0}-y\right)^{2} / 4(1-t)} \phi(y) d y,
\end{aligned}
$$

provided we justify the interchange of summation and integration.

For this it suffices to show that

$$
K\left(x_{0}, t\right)=(4 \pi)^{-\frac{1}{2}} \sum_{r=0}^{\infty} t^{r} \int_{-\infty}^{\infty} e^{-\frac{1}{4}\left(x_{0}-y\right)^{2}}\left|L_{r}^{-\frac{1}{2}}\left(\frac{1}{4}\left(x_{0}-y\right)^{2}\right) \phi(y)\right| d y
$$

is finite. But from $(3 ; 10.18(15))$ it follows that

$$
\left|L_{n}^{-\frac{1}{2}}(x)\right| \leqslant 2 e^{\frac{3}{2} x}
$$

so that

$$
K\left(x_{0}, t\right) \leqslant \frac{1}{\pi^{\frac{1}{2}}(1-t)} \int_{-\infty}^{\infty} e^{-\left(x_{0}-y\right)^{2} / 8}|\phi(y)| d y<\infty
$$

for $0 \leqslant t<1$, and the interchange is justified.

Hence if $0 \leqslant t<1, \delta>0$,

$$
\begin{aligned}
u\left(x_{0}, t\right) & =\frac{1}{(4 \pi(1-t))^{\frac{1}{2}}} \int_{-\infty}^{\infty} e^{-\left(x_{0}-y\right)^{2} / 4(1-t)} \phi(y) d y \\
& =\int_{-\infty}^{x_{0}-\delta}+\int_{x_{0}-\delta}^{x_{0}+\delta}+\int_{x_{0}+\delta}^{\infty}=I_{1}+I_{2}+I_{3} .
\end{aligned}
$$

Consider $I_{3}$. Since $e^{-\left(x_{0}-y\right)^{2} t / 4(1-t)}$ decreases for $y>x_{0}$, we have

$$
\begin{aligned}
\left|I_{3}\right| & \leqslant \frac{1}{(4 \pi(1-t))^{\frac{\pi}{2}}} \int_{x_{0}+\delta}^{\infty} e^{-\left(x_{0}-y\right)^{2} / 4(1-t)}|\phi(y)| d y \\
& =\frac{1}{(4 \pi(1-t))^{\frac{1}{2}}} \int_{x_{0}+\delta}^{\infty} e^{-\left(x_{0}-y\right)^{2} t / 4(1-t)} e^{-\frac{1}{2}\left(x_{0}-y\right)^{2}}|\phi(y)| d y \\
& \leqslant \frac{e^{-\delta^{2} t / 4(1-t)}}{(4 \pi(1-t))^{\frac{1}{2}}} \int_{-\infty}^{\infty} e^{-\frac{1}{t}\left(x_{0}-y\right)^{2}}|\phi(y)| d y \rightarrow 0 \quad \text { as } t \rightarrow 1-
\end{aligned}
$$

Similarly $I_{1} \rightarrow 0$ as $t \rightarrow 1-$.

Finally from (7; chap. VII, Theorem $2 \mathrm{~b}$ and corollaries $2 \mathrm{~b} .2$ and $2 \mathrm{~b} .3$ ), $I_{2} \rightarrow \frac{1}{2}\left\{\phi\left(x_{0}+\right)+\phi\left(x_{0}-\right)\right\}$.

Corollary. If

$$
e^{-\left(x_{0}-t\right)^{2} / 8} \phi(t) \in L(-\infty, \infty)
$$

and $x_{0}$ is in the Lebesgue set of $\phi$, then $\mathscr{G}(\phi(x))$ exists for all $x$, and the series II is Abel summable for $x=x_{0}$ to $\phi\left(x_{0}\right)$.

The proof is identical with the proof of the theorem except that we do not use (7; Chap. VII, Corollary 2b.3). 
It may be mentioned that

$$
\frac{e^{t^{2} / 8}}{1+t^{2}}
$$

provides, with $x_{0}=0$, an example of a function satisfying the hypotheses of Theorem 2, but not those of Theorem 1 .

4. Applications. Theorem 1 yields various results about Hermite polynomials. For example, it follows from $(3 ; 10.13(30))$ that

$$
\mathscr{G}\left(H_{n}(a x)\right)=\left(1-4 a^{2}\right)^{\frac{1}{2} n} H_{n}\left(a x /\left(1-4 a^{2}\right)^{\frac{1}{2}}\right), \quad-\frac{1}{2}<a<\frac{1}{2} .
$$

Now from $(3 ; 101.3(13))$,

$$
D^{r} H_{n}(b x)= \begin{cases}(2 b)^{r} \cdot n(n-1) \ldots(n-r+1) H_{n-r}(b x), & r \leqslant n \\ 0 & r>n .\end{cases}
$$

Hence from Theorem 1 we have, if $-\frac{1}{2}<a<\frac{1}{2}$,

$$
\begin{aligned}
H_{n}(a x)=\left(1-4 a^{2}\right)^{\frac{1}{2} n} \sum_{r=0}^{\left[\frac{1}{2} n\right]} \frac{n(n-1) \ldots(n-2 r+1)}{r !} \\
\cdot\left(-4 a^{2} /\left(1-4 a^{2}\right)\right)^{r} H_{n-2 r}\left(a x /\left(1-4 a^{2}\right)^{\frac{1}{2}}\right),
\end{aligned}
$$

or changing $a x /\left(1-4 a^{2}\right)^{\frac{1}{2}}$ to $x$, and letting $\lambda=\left(1-4 a^{2}\right)^{\frac{1}{2}}$,

$$
H_{n}(\lambda x)=\sum_{r=0}^{\left[\frac{1}{2} n\right]} \frac{n(n-1) \ldots(n-2 r+1)}{r !}\left(\lambda^{2}-1\right)^{r} \lambda^{n-2 r} H_{n-2 r}(x),
$$

the result being clearly valid now for all $\lambda$.

Another interesting application comes about as follows. An easy calculation shows that for all real $v$,

$$
\text { (1) }\left(\left(1+4 v^{2}\right)^{-\frac{1}{2}} e^{v^{2} x^{2} /\left(1+4 v^{2}\right)}\right)=e^{v^{2} x^{2}}=f(x),
$$

and it follows easily from differentiating this formula that

$$
\mathscr{G}\left(\left(1+4 v^{2}\right)^{-3 / 2} x e^{\eta^{2} x^{2} /\left(1+4 x^{2}\right)}\right)=x e^{v^{2} x^{2}}=g(x) .
$$

Theorem 1 can be applied to these functions if $-\frac{1}{2}<v<\frac{1}{2}$. Hence since, from $(3 ;(10.13(7))$

$$
f^{(2 r)}(x)=D^{2 r} e^{v^{2} x^{2}}=(-1)^{r} v^{2 r} e^{v^{2} x^{2}} H_{2 \tau}(i v x),
$$

and using $(3 ; 10.13(10))$ and Leibnitz's rule,

$$
\begin{aligned}
g^{(2 r)}(x) & =D^{2 r} x e^{v^{2} x^{2}}=x D^{2 r} e^{v^{2} x^{2}}+2 r D^{2 r-1} e^{v^{2} x^{2}} \\
& =(-1)^{r} v^{2 r-1} e^{v^{2} x^{2}}\left(2 i v x H_{2 r}(i v x)-4 r H_{2 r-1}(i v x)\right) / 2 \imath \\
& =(-1)^{\tau} v^{2 r-1} e^{v^{2} x^{2}} H_{2 r+1}(i v x) / 2 i .
\end{aligned}
$$

Thus from Theorem 1 , if $-\frac{1}{2}<v<\frac{1}{2}$, 


$$
\left(1+4 v^{2}\right)^{-\frac{1}{2}} e^{v^{2} x^{2} /\left(1+4 v^{2}\right)}=e^{v^{2} x^{2}} \sum_{r=0}^{\infty} v^{2 r} H_{2 r}(i v x) / r !
$$

and

$$
\left(1+4 v^{2}\right)^{-3 / 2} x e^{v^{2} x^{2} /\left(1+4 v^{2}\right)}=\left(2 i v^{2}\right)^{-1} e^{v^{2} x^{2}} \sum_{r=0}^{\infty} v^{2 r+1} H_{2 r+1}(i v x) / r !,
$$

whence on dividing we obtain

$$
\left(1+4 v^{2}\right)^{-\frac{1}{2}} e^{4 v^{4} x^{2} /\left(1+4 v^{2}\right)}=\sum_{r=0}^{\infty} v^{2 r} H_{2 r}(i v x) / r !
$$

and

$$
\left(1+4 v^{2}\right)^{-3 / 2} \cdot 2 i v^{2} x e^{4 v^{4} x^{2} /\left(1+4 v^{2}\right)}=\sum_{r=0}^{\infty} v^{2 r+1} H_{2 r+1}(i v x) / r !
$$

Now for every $v,-\frac{1}{2}<v<\frac{1}{2}$, each series of the last pair converges uniformly with respect to $x$ for $x$ in any closed bounded region of the complex $x$-plane. This follows for the first series, for example from the $M$-test on making use of the inequality

$$
\left|H_{2 r}(x)\right| \leqslant 2^{2 r+1} r ! e^{\frac{1}{2} x^{2}}
$$

given in $(3 ; 10.18(16))$, and for the second series similarly. Hence by the principal of analytic continuation, the series converge to the indicated sums for all complex $x$, and another appeal to analytic continuation with respect to $v$ shows that the formulae hold for all complex $v$ with $|v|<\frac{1}{2}$. Finally,

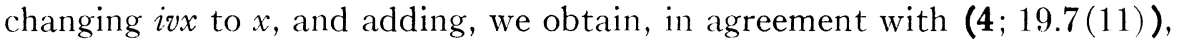

$$
\frac{1+2 v x+4 v^{2}}{\left(1+4 v^{2}\right)^{3 / 2}} e^{4 v^{2} x^{2} /\left(1+4 v^{2}\right)}=\sum_{n=0}^{\infty} v^{n} H_{n}(x) /\left[\frac{1}{2} n\right] !
$$

valid for all complex $x$, and all complex $v$ with $|v|<\frac{1}{2}$.

\section{REFERENCES}

1. G. Doetsch, Handbuch der Laplace-Transformation (Basel, 1950).

2. A. S. Eddington, On a formula for correcting statistics for a known probable error of observation, Monthly Notices Roy. Astron. Soc., 73 (1914), 359-360.

3. A. Erdélyi, et al., Higher Transcendental Functions, vol. 2 (New York, 1953).

4. - Higher Transcendental Functions, vol. 3 (New York, 1953).

5. I. I. Hirschmann and D. V. Widder, The Convolution Transform (Princeton, 1955).

6. H. Pollard, Integral transforms, Duke Math. J., 13 (1946), 307-330.

7. D. V. Widder, The Laplace Transform (Princeton, 1941).

8. - Necessary and sufficient conditions for the representation of a function by a Weierstrass transform, Trans. Amer. Math. Soc., 71 (1951), 430-439.

\section{University of Toronto}

